\title{
E-Band Beam-Steerable and Scalable Phased Antenna Array for 5G Access Point
}

\author{
Md. Mazidul Islam (D, ${ }^{1}$ Mikko Leino, ${ }^{2}$ Rasmus Luomaniemi, ${ }^{2}$ Jinsong Song, ${ }^{3}$ \\ Risto Valkonen, ${ }^{4}$ Juha Ala-Laurinaho $\mathbb{D}^{2},{ }^{2}$ and Ville Viikari ${ }^{2}{ }^{2}$ \\ ${ }^{1}$ Keysight Technologies Finland Oy, Elektroniikkatie 10, 90590 Oulu, Finland \\ ${ }^{2}$ Department of Electronics and Nanoengineering, Aalto University, Aalto FI-00076, Finland \\ ${ }^{3}$ Smart Antenna Technologies Ltd., Birmingham Research Park, Birmingham B15 2SQ, UK \\ ${ }^{4}$ Nokia Bell Labs, Nokia, Espoo 00045, Finland \\ Correspondence should be addressed to Md. Mazidul Islam; md-mazidul.islam@keysight.com
}

Received 9 April 2018; Revised 29 August 2018; Accepted 17 September 2018; Published 28 November 2018

Academic Editor: Chien-Jen Wang

Copyright (c) $2018 \mathrm{Md}$. Mazidul Islam et al. This is an open access article distributed under the Creative Commons Attribution License, which permits unrestricted use, distribution, and reproduction in any medium, provided the original work is properly cited.

\begin{abstract}
This paper presents a new implementation of the beam-steerable two-dimensional phased antenna array for the forthcoming $5 \mathrm{G}$ networks. The antenna enables easy integration of phase shifters and other active electronics on a single PCB, low-loss feed network, low profile, and beam steering in both azimuth and elevation plane. In addition, the antenna is scalable in the number of elements and it can be made compatible with low-cost mass production in plastic injection molding with a metal coating. The antenna consists of a rectangular waveguide feed network, waveguide-to-PCB transitions, phase shifters on a PCB, and horn antenna radiating elements. The parts have been first designed and simulated individually and the operation of the whole structure is then verified by electromagnetic simulations. The phase shifter used in this work is a meandered microstrip line section, but the structure also enables the implementation of active phase shifters. A four-by-four antenna array prototype was manufactured. The beam-steering properties of the phased antenna array have been tested with radiation pattern measurements at $72.5 \mathrm{GHz}$, and the measured gains are compared with the simulated ones. The measured gains are 15.2 and $11.2 \mathrm{dBi}$ for the boresight beam, and the beam was steered to $40^{\circ}$.
\end{abstract}

\section{Introduction}

Millimeter-wave link frequencies such as 28,38 , and $60 \mathrm{GHz}$, as well as the E-band (71-76 and $81-86 \mathrm{GHz})$, have been proposed for use in wireless backhaul infrastructure to enable 5G networks [1-6]. Especially, the E-band provides a huge spectral resource compared to traditional microwave bands $(6-42 \mathrm{GHz})$ and bandwidths comparable to $10-100 \mathrm{~Gb} / \mathrm{s}$ fiber connections with low latency.

Electrical beam steering in two dimensions, that is, in azimuth and in elevation is required to ease the antenna installation and to cope with the $5 \mathrm{G}$ small cell deployment sites during the operation [7]. Additionally, antennas need to be small and have a low profile for imperceptible installations. High-gain antennas are needed together with a possibility to easily accommodate active components, such as phase shifters and amplifiers, to overcome propagation losses at mm-wave frequencies, increase coverage area, and improve system performance. The solution is ideally scalable in the number of elements for different antenna gains.

There are many approaches proposed for implementing electrical beam-steering antennas, including beam switching/beam selection networks, reconfigurable highimpedance surfaces (HIS), and phase shifting or timedelay networks. An integrated lens antenna (ILA) provides good beam-steering characteristics both in the azimuth and elevation planes $[7,8]$. For each beam, there is a corresponding separate element in the focal feed array. For this purpose, a complex switching network is needed. Each switch has a considerable attenuation at mm-waves from 2 to $3 \mathrm{~dB}$ resulting in high overall attenuation of the switching network. Another7 drawback is that the lens is bulky. 


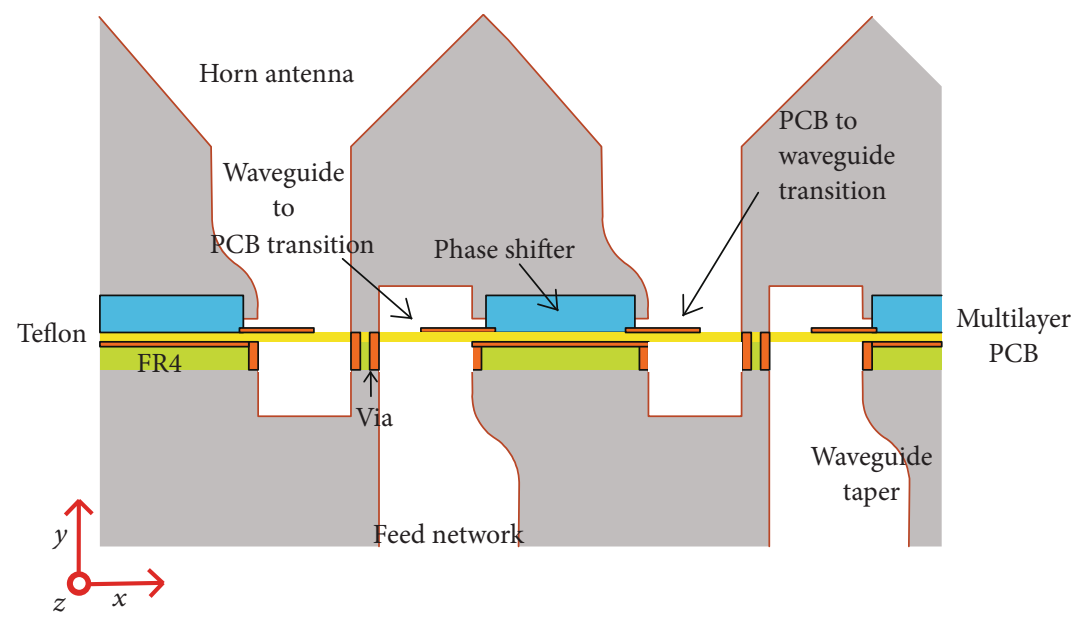

FIGURE 1: Cutaway picture of a small section of the proposed phased horn antenna array from the side. The feed network is realized with waveguides and only the output waveguides of the feed network are shown. Phase shifters are realized on a multilayer PCB.

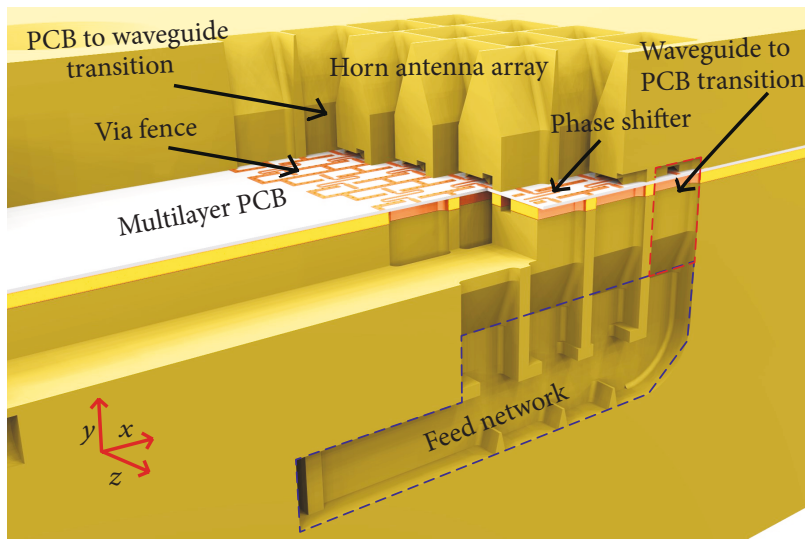

FIGURE 2: 3D model cutaway picture of the proposed phased antenna array.

Beam switching is also used in passive beamforming networks, such as the Rotman lens [9] or Butler matrix [10]. A complete $60 \mathrm{GHz}$ beam-switching antenna based on a Rotman lens is presented in [11]. The complexity of the Rotman lenses increases rapidly with the number of the input ports. The design tradeoff of the switching network is similar to that of the ILAs. A larger number of beams can result in an increased directivity or wider angular coverage, but at the expense of a larger number of switches and higher switching losses. Similar to a conventional lens, the Rotman lens and Butler matrix are bulky, especially when they are used for two-dimensional beam steering.

At millimeter-wave frequencies, the reflectarray has the advantage of being light weight. Reconfigurable reflectarrays are presented, for example, in $[12,13]$. However, a separate feed antenna is needed which increases the antenna volume. To overcome this problem, a folded reflectarray solution is proposed [14]. Reflectarray avoids some of the feed network losses associated to beam-switchable lenses or phased arrays, but makes it challenging to add phase shifters. Recently, conventional lenses and reflectarray-type antennas are

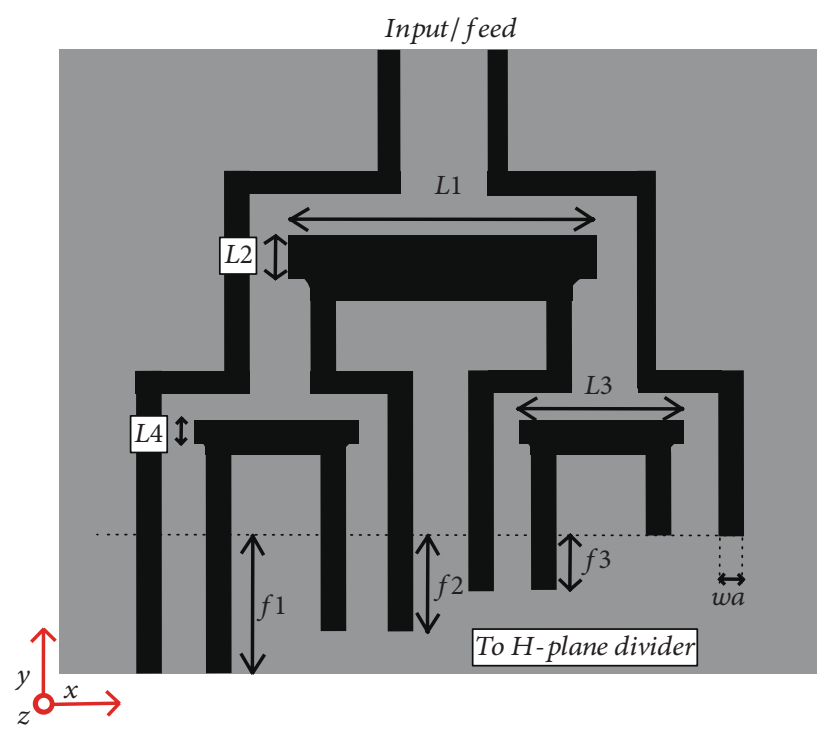

FIGURE 3: Internal structure of the primary feed divider.

realized using metamaterials [15-20]. Although some broadband solutions are proposed, most metamaterials are based on resonant structures and typically have a narrow band.

An alternative approach for beam steering is to use phased antenna arrays. In full digital beam forming [21], full RF chains and data converters for every antenna element are required together with heavy processing capability. Thus, it is complex, expensive, and power hungry at high frequencies. Recently, several interesting scalable phased array structures and designs for $\mathrm{mm}$-wave frequencies have been proposed $[22,23]$. A major challenge in low-profile high-gain millimeter-wave antennas is the high losses in the feed network because the transmission lines realized on $\mathrm{PCB}$ become relatively long and they have inherently high losses at high frequencies. In addition, passive reconfigurable phase shifters also introduce losses of many $\mathrm{dBs}[24,25]$. Also, unwanted grating lobes may appear during 2-D 


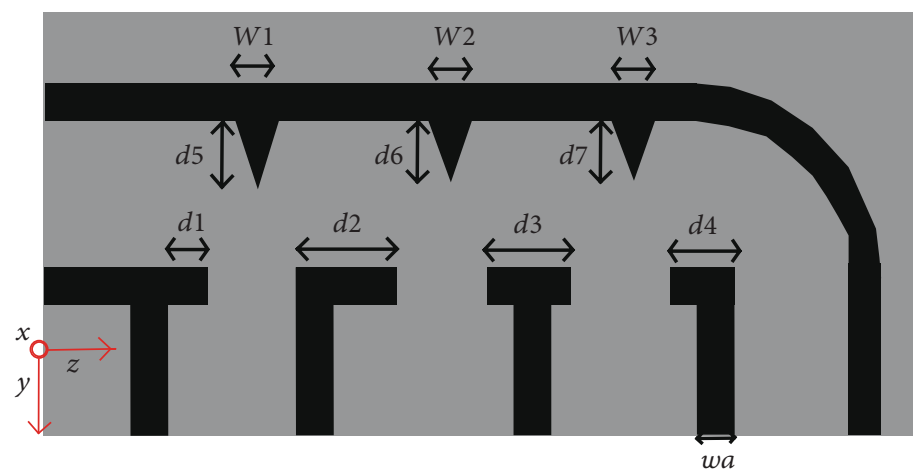

FIGURE 4: Internal structure of the secondary feed divider.

beam steering because of the limitations for the element spacing in the antenna array.

Plastic injection molding is an effective way to produce millimeter-wave antennas with a flat design, low weight, and a competitive price. In this manufacturing technology, mechanical tolerances can be controlled very tightly and complex three-dimensional shapes can be realized in high quantities. Plastic injection molding techniques with application to the realization of millimeter-wave components have been investigated thoroughly in $[26,27]$. The technology is mature, and plastic injection molding antennas are commercially available, such as the Nokia MetroHopper (which is based on the work published in $[28,29]$ ) and Huber + Suhner SENCITY Matrix [30]. However, state-of-the-art plastic injection molded antennas do not accommodate active electronics that enable it to perform electronic beam steering.

As we discussed earlier, all the previously presented antenna types have some inconveniences. No single antenna type can simultaneously exhibit all the desired features for $5 \mathrm{G}$ networks. In this paper, we present a phased antenna array structure potentially capable of offering all the benefits at the lower E-band $(71-76 \mathrm{GHz})$, namely, low loss, easy integration of active electronics, scalability, and wide 2-D beam-steering range. Moreover, our proposed antenna could be manufactured using low-cost plastic injection molding with a metal coating. For the first time, we present a plastic injection moldable structure accommodating phase shifters and possibly other active electronics on a single PCB.

The proposed phased antenna array consists of a rectangular waveguide power divider structure, waveguide-to-PCB transitions, microstrip phase shifters, and pyramidal horns as antenna elements. In this work, a 4-by-4 phased antenna array is designed, simulated, and verified with the measured results of the fabricated prototype. Figures 1 and 2 show cutaway pictures of the proposed antenna where the feed network is realized with rectangular waveguides for minimizing the losses. Meandered sections of a microstrip line are used as phase shifters in this work. For an actual beam-steering application, active phase shifters should be implemented. Excluding the PCB board, the parts of the proposed antenna structure can be manufactured with plastic injection molding followed by metallization which enables a cost-efficient manufacturing process.
TABLE 1: Optimized dimensions of the waveguide feed network (all dimensions are in $\mathrm{mm}$ ).

\begin{tabular}{lccccccc}
\hline$L 1$ & $L 2$ & $L 3$ & $L 4$ & $d 1$ & $d 2$ & $d 3$ & $d 4$ \\
\hline 6 & 0.85 & 2.52 & 0.64 & 0.87 & 1.17 & 0.85 & 0.98 \\
$d 5$ & $d 6$ & $d 7$ & $W 1$ & $W 2$ & $W 3$ & a (inner) & b (inner) \\
0.84 & 0.84 & 0.91 & 0.5 & 0.51 & 0.52 & 2.7 & 1.35 \\
\hline
\end{tabular}

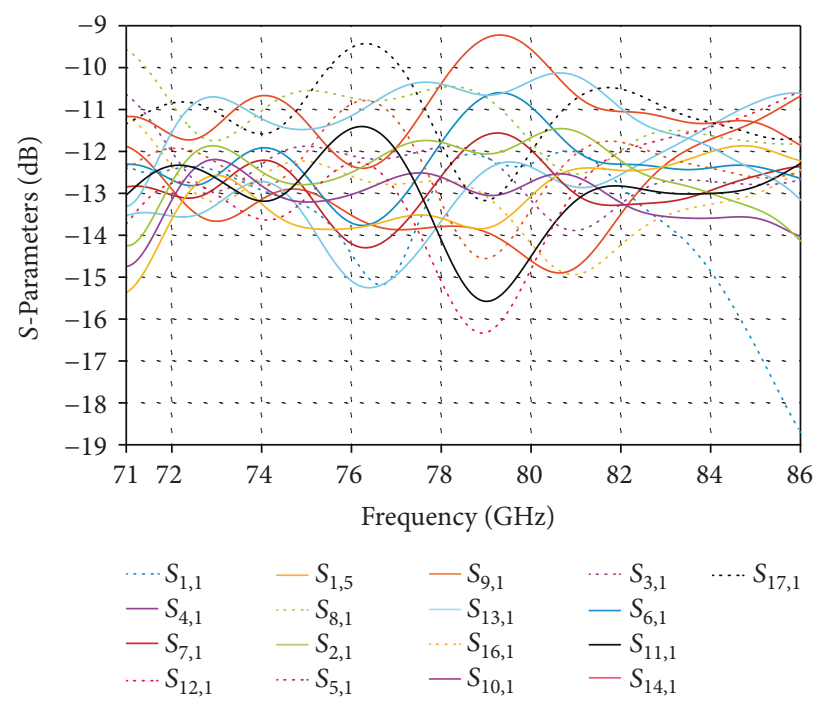

Figure 5: Simulated S-parameters of the waveguide feed network.

This paper is organized as follows. Section 2 describes the key components of the antenna array: waveguide power division network, waveguide-to-PCB transitions, phase shifters, and horn antenna elements. Section 4 shows the measurement results of the fabricated prototype. The use of the proposed antenna concept in the $5 \mathrm{G}$ scenario is discussed in Section 4, and the conclusions are drawn in Section 5.

\section{Design of Key Components of the mm-Wave Antenna Array}

The design of the key antenna components is presented in Sections 2.1-2.4. The parts are designed using CST 


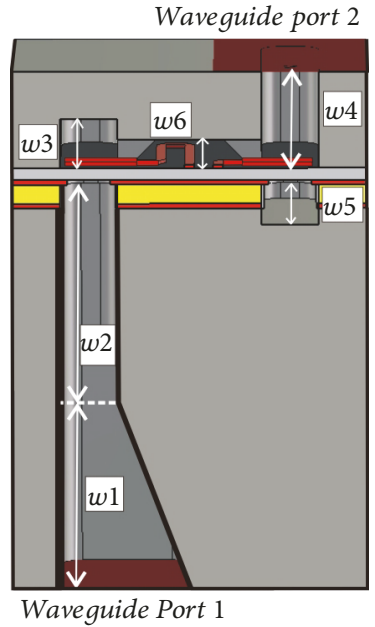

(a)

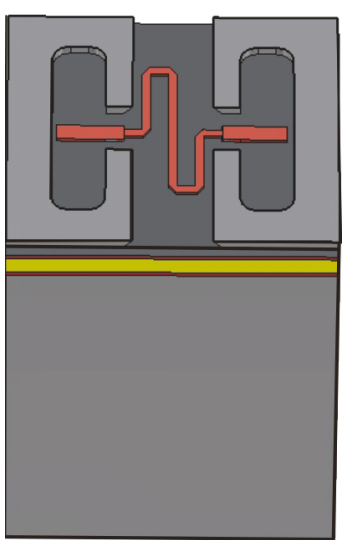

(b)

Figure 6: Simulation model of the waveguide-to-PCB transition, phase shifter, and the PCB-to-waveguide transitions. (a) Side-cut view and (b) top-cut view. Although the transitions look the same, there are differences between them because they are located on opposite sides of the PCB.

electromagnetic simulation software. Brass with $65 \%$ copper content (conductivity $\sigma=5.9 \times 10^{6} \mathrm{~S} / \mathrm{m}$ ) is used for the metals in the simulation.

2.1. Power Division Network. The power divider is designed to provide uniform signal strength for each horn antenna element with as low power division losses as possible. Figures 3 and 4 show the two main parts of the waveguide feed network. The primary feed divider consists of three E-plane Tjunctions combined together. The four secondary dividers are formed from three $\mathrm{H}$-plane $\mathrm{T}$-junctions. Table 1 presents the optimized values for the dimensions presented in Figures 3 and 4 .

The antenna is fed with a standard WR-12 waveguide $\left(3.1 \times 1.55 \mathrm{~mm}^{2}\right)$. The desired spacing between the elements is $\lambda / 2$ at the highest operation frequency to avoid grating lobes at all beam-steering angles. However, due to the size of the feeding waveguide, finite wall thickness between adjacent waveguides, and space needed for the phase shifters, spacing larger than $\lambda / 2$ had to be used. Moreover, although the antenna structure is designed to be plastic injection moldable, the prototype is manufactured by milling for lower prototyping costs. This manufacturing process also poses some additional limitations to the structure such as a minimum wall thickness of $w a=0.5 \mathrm{~mm}$ and an inner radius of the bending of $0.2-0.5 \mathrm{~mm}$ depending on the milling tools. The element spacing is set to the smallest possible value, which is about $0.8 \lambda$ at $75 \mathrm{GHz}$ in this case. Thus, the grating lobes start to arise when the beam is steered. For a uniformly spaced array, the first grating lobe appears when the beam is steered about $14^{\circ}$ and, eventually, the grating lobe equals the main beam in amplitude for the steering angle of $38^{\circ}$. The inner dimensions of the rectangular waveguide in the feed network is set to $2.7 \times 1.35 \mathrm{~mm}^{2}$. The cutoff frequency for a waveguide with these dimensions is $55 \mathrm{GHz}$ which is sufficiently low to ensure low-loss operation at the lower Eband $(71-76 \mathrm{GHz})$.

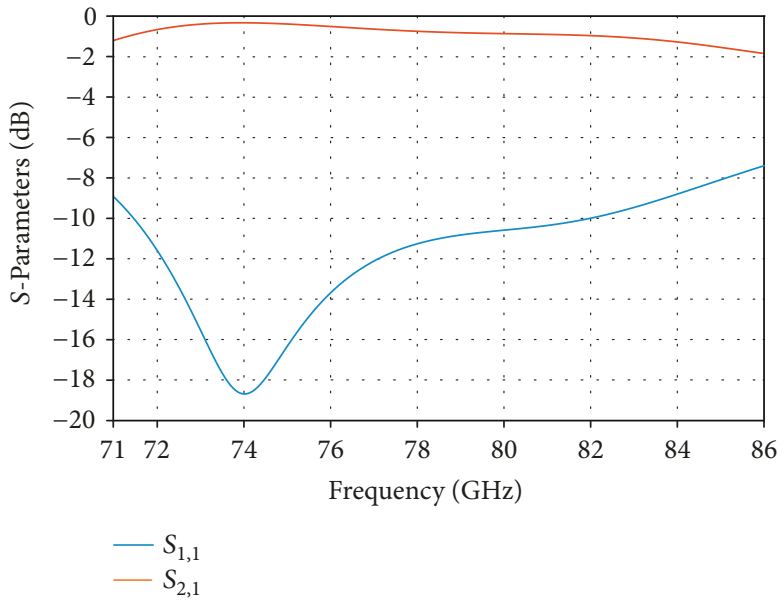

FIGURE 7: Simulated $S$-parameters of the ensemble consisting of the waveguide-to-PCB transition, phase shifter $(x=0.5 \mathrm{~mm})$, and the PCB-to-waveguide transition (see Figure 6).

2.1.1. Primary Feed Divider. The purpose of the primary feed divider is to divide the power equally from the input waveguide into four output waveguides. The primary feed divider consists of three power splitters from which two are identical. The first power splitter divides the power equally between the two output branches. Both output branches are followed by the second power splitters, such that the original signal is split into four. The dimension of the $L 1, L 3$, and matching steps ( $L 3$ and $L 4$ ) all contribute to the final impedance matching and power ratio between the ports.

The final inner structure of the primary feed divider is shown in Figure 3. Ports $(f 1=2.4 \mathrm{~mm}, f 2=1.6 \mathrm{~mm}$, and $f 3=0.8 \mathrm{~mm}$ ) are slightly offset, as shown in Figure 3 . The element locations are slightly offset to avoid grating lobes in the main pattern cut (along $\theta$ ). 


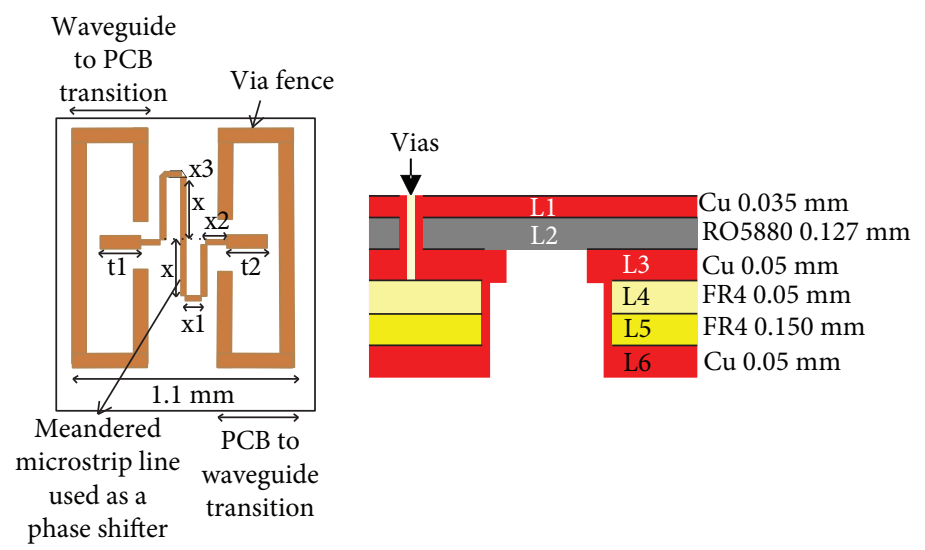

(a)

(b)

FIGURE 8: (a) The PCB structure consisting of the waveguide-to-PCB transition, phase shifter, and PCB-to-waveguide transition. (b) Layer stack-up for manufacturing the multilayer PCB.

2.1.2. Secondary Feed Divider. The purpose of the secondary feed divider is to subdivide each of the four output channels from the primary divider into 4 ports, so that the primary and secondary dividers split the signals into 16 ports. This secondary feed divider is formed by a combination of $\mathrm{H}$ plane T-junctions. Proper matching and power division has been achieved by tuning the size and position of the metal ridges and the coupling aperture dimensions of the output waveguides $(d 1-d 7, W 1-W 3)$. Note that there is a phase difference between the ports which finally has to be compensated with phase shifters.

Figure 4 shows the final inner structure of the secondary feed branch. Figure 5 shows the $S$-parameters of the waveguide feed network. Parameters are tuned to achieve the best performance at the lower E-band $(71-76 \mathrm{GHz})$. The insertion loss between the branches varies between 9 and $15 \mathrm{~dB}$.

\subsection{Waveguide-to-PCB Transitions and Phase Shifter. There} are two different waveguide-to-PCB transitions in the antenna structure. One is from the power divider output waveguide to the PCB and the other is from the PCB to the antenna waveguide. At the transition, the waveguides are tapered to half of the height of the original waveguides to have enough space for the phase shifters. This also helps in impedance matching. The PCB cuts through the waveguide and the microstrip line on the top and enters the waveguide through a small opening, thus forming a probe. In addition, the walls of the waveguides are beveled so that there is more space for the phase shifter. Figure 6 shows the simulation model of the waveguide-to-PCB transition, phase shifter, and the PCB-to-waveguide transition. Figure 7 shows the simulated $S$-parameters for the structure, including both transitions and the phase shifter. The reflection coefficient is well below $-10 \mathrm{~dB}$ almost in the full lower E-band. The phase shifter length $x=0.5 \mathrm{~mm}$ is used in this simulation.

Figure $8(\mathrm{~b})$ shows the multilayered PCB stack-up of RO5880 $\left(\epsilon_{r}=2.20\right.$ and $\left.\tan \delta=0.0009\right)$, and FR4 is used for the phase shifter implementation. Vias are made between $L 1$ and $L 3$ to form a waveguide through the RO5880 layer, whereas a cavity is processed in the FR-4 layer
TABle 2: Optimized dimensions for the waveguide-to-PCB transitions, and the phase shifter (all dimensions are in $\mathrm{mm}$ ).

\begin{tabular}{lccccc}
\hline$t 1$ & $t 2$ & $x$ & $x 1$ & $x 2$ & $x 3$ \\
\hline 0.748 & 0.72 & $0.2-0.95$ & 0.2 & 0.22 & 0.14 \\
$w 1$ & $w 2$ & $w 3$ & $w 4$ & $w 5$ & $w 6$ \\
2.08 & 2.26 & 0.42 & 1.04 & 0.48 & 0.3 \\
\hline
\end{tabular}

between $L 3$ and $L 6$ around the waveguide. Finally, edge metallization is applied to the cavity surface to ensure grounding between $L 3$ and $L 6$. Table 2 presents the optimized dimensions of the transitions. The E-band phase shifter is realized with a meandered microstrip line section in layer $L 1$ (see Figure $8(\mathrm{a})$ ). The length of the microstrip line is adjusted according to the required time delay or phase change. The microstrip line is realized on top of the RO5880 layer, and an FR4 layer below simulates a layer for carrying digital control signals and supplies voltage to the phase shifters that could also be of the active type. A cavity is milled above the phase shifter for integration in the antenna structure (see Figure 6). Figure 9 shows the amplitude of the reflection and transmission coefficients of the phase shifter for three different values of $x$.

In this work, several PCB assemblies, each containing a set of 16 phase shifters, are designed to correspond to predefined beam-steering angles. Discrete phase shifters for 32 different $x$ values are designed corresponding to a 5 -bit phase shifter. These phase shift values are used once defining the PCB assemblies. Phases of $S_{21}$ of the phase shifters as a function of $x$ (the length of the phase shifter) are shown in Figure 10.

2.3. Horn Antenna. The radiating elements are pyramidal horn antennas, see Figure 2. The horn aperture size is $3.1 \mathrm{~mm} \times 3.1 \mathrm{~mm}$ which is mostly defined by the waveguide dimensions. The width of the waveguide and the wall thickness between adjacent elements determine the aperture size 


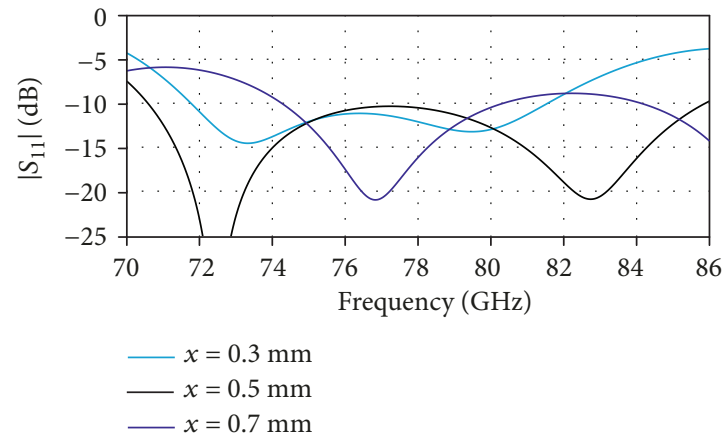

(a)

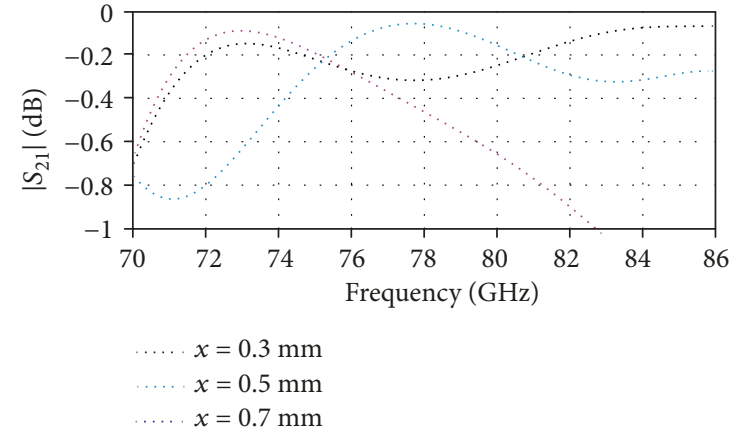

(b)

FIgURE 9: Simulated $S$-parameters of the phase shifter, (a) $S_{11}$ and (b) $S_{21}$.

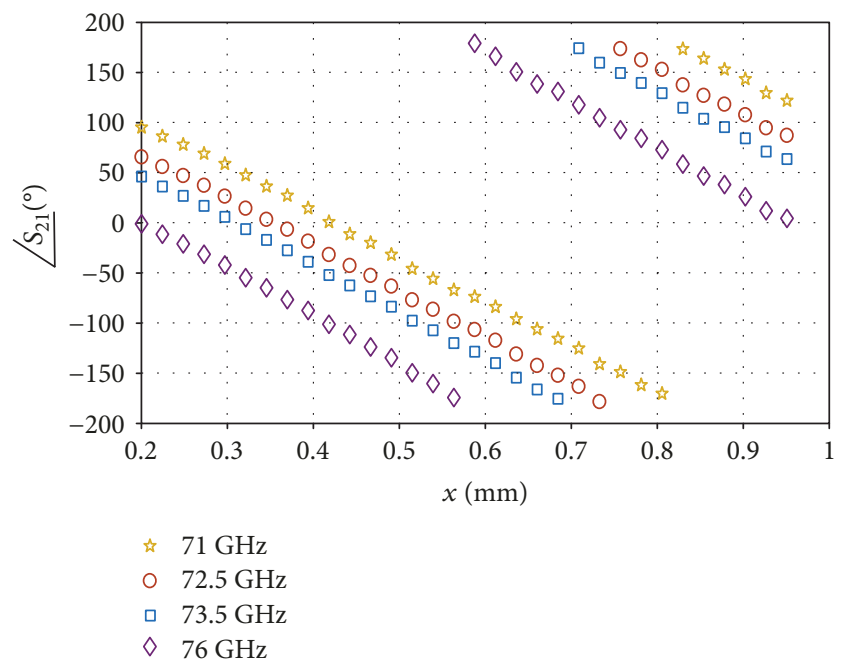

Figure 10: Simulated phase of the $S_{21}$ of the phase shifter as a function of $x$.

in the E-plane. The aperture size in the H-plane is determined by the symmetry. A $0.1 \mathrm{~mm}$-wide ridge is left between the adjacent horn antennas. The aperture is smoothly tapered towards the lowered waveguide of the PCB-to-waveguide transition. The length of the horn antenna is $4.08 \mathrm{~mm}$.

2.4. Full Antenna Array. Figure 2 shows the model of the 4by- 4 phased antenna array. The final simulation model of the full antenna array is made by integrating all of the key components. The simulated reflection coefficient under the boresight directions is shown in Figure 11. The simulated reflection coefficient is below $-10 \mathrm{~dB}$ almost in the whole desired frequency range.

\section{Prototype Fabrication and Measurement Results}

The prototypes of the feed network and antennas have been manufactured by milling the structures in brass $\mathrm{CuZn} 39 \mathrm{~Pb} 3$ $(\mathrm{CW} 614 \mathrm{~N})$ with a copper content of $57-59 \%$, thus the conductivity might be slightly lower than that used in the

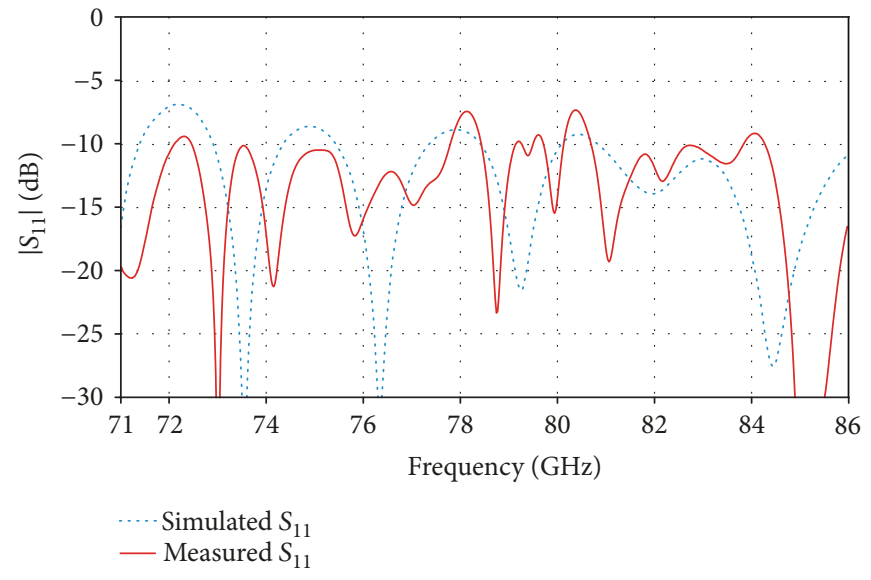

Figure 11: Measured and simulated $S_{11}$ for the boresight.

simulation. Transitions and phase shifters have been realized on a multilayer printed-circuit board (PCB). Figure 12 shows the assembled prototype antenna and one fabricated phase shifter PCB. The far-field pattern of the antenna is determined by near-field measurement. The near-field is measured at a distance of $30 \mathrm{~mm}$ with an open-ended WR-10 waveguide as the probe. The measurement setup is shown in Figure 13. The probe compensation is applied based on the measured properties of the used probe. The gain is determined by comparison with a reference horn antenna.

Even though the antenna is designed to demonstrate the beam-steering properties at a $73.5 \mathrm{GHz}$ center frequency, the measured center frequency response of the antenna was observed at a slightly lower frequency of $72.5 \mathrm{GHz}$ due to a manufacturing error. There is a small milling error; that is, the height of the last matching step $(d 7)$ and the height each step are too low by $0.07 \mathrm{~mm}$. However, this discrepancy does not change much the beam-steering properties of the antenna. The antenna is tested using 11 different phase shifter printed-circuit boards (PCB) at $72.5 \mathrm{GHz}$. Each PCB corresponds to a different beam-steering angle.

Figure 11 shows the measured and simulated reflection coefficient $\left(S_{11}\right)$ for the phased antenna array pointing towards the boresight direction. The reflection coefficients match well and the measured reflection coefficient is almost 


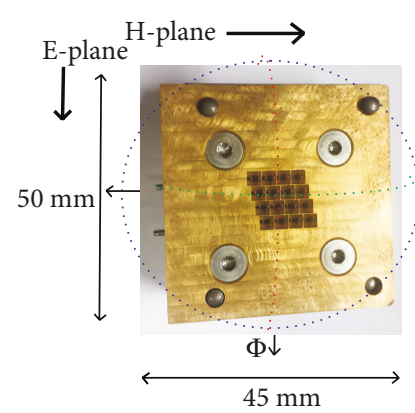

(a)

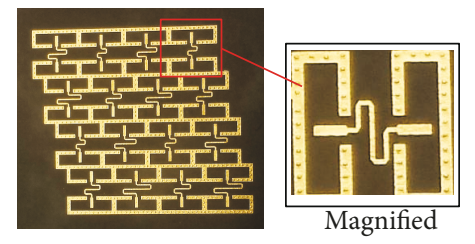

(b)

FIGURE 12: (a) The assembled antenna and (b) phase shifter printed-circuit boards at $72.5 \mathrm{GHz}$.

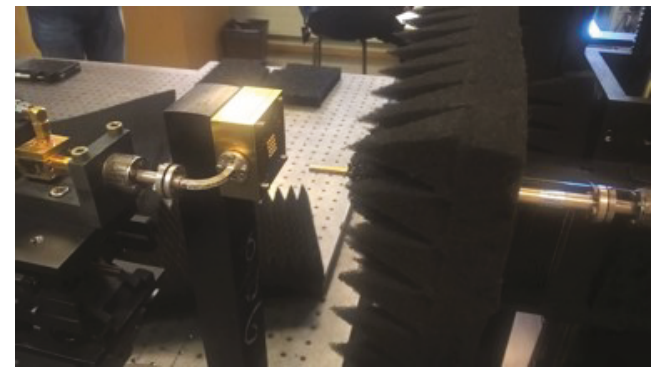

FIGURE 13: Assembled antenna in the measurement setup.

below $-10 \mathrm{~dB}$ in the whole E-band $(71-86 \mathrm{GHz})$. In addition, the measured reflections for the different beam-steering angles are shown in Figure 14. The measured and simulated radiation patterns in both elevation and azimuth plane, that is, the E-plane and H-plane, for the phased antenna array pointing toward the boresight direction are shown in Figure 15 . The measured radiation pattern agrees reasonably well with the simulated one. Furthermore, Figure 16 shows the measured and simulated 2-dimensional radiation patterns at $72.5 \mathrm{GHz}$ for the same antenna assembly.

Table 3 summarizes the simulated and measured antenna characteristics for different beam-steering angles at $72.5 \mathrm{GHz}$. Here, the measured antenna gain and half-power beam width for different beam-steering angles match well with the simulation results. Based on the result, the proposed antenna structure has an approximately $5 \mathrm{~dB}$ beam scanning loss at $40^{\circ}$ in both planes. The measured antenna gain is $15.2 \mathrm{dBi}$ and the half-power beam width is $18.4^{\circ}$, whereas the simulated values are $16.9 \mathrm{dBi}$ and $18.6^{\circ}$, respectively, when the beam points towards the boresight. In the prototype, the used brass conductivity is probably slightly different from the one used in the simulations which may lead to a slightly lower gain in the measurements.

As an example of the eleven assemblies, the radiation patterns for the four beam-steering angles of $10^{\circ}, 20^{\circ}, 30^{\circ}$, and $40^{\circ}$ in both elevation and azimuth plane, are shown in Figures 17 and 18, respectively. As can be seen from Figure 17, a beam steers well in the elevation plane. However, the grating lobe appears for the $30^{\circ}$ beam-steering angle, and for the $40^{\circ}$ beam-steering angle the grating lobe is at a slightly

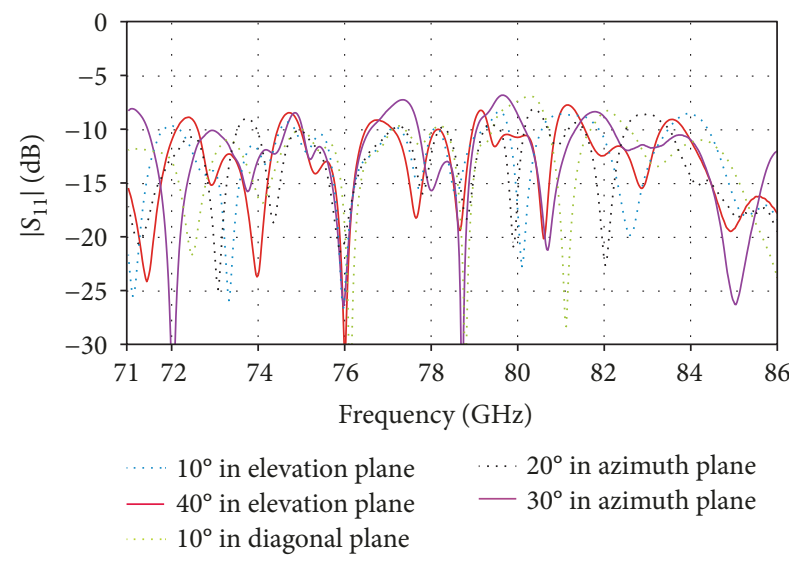

Figure 14: Measured reflection coefficient for different beamsteering angles.

higher level than the desired beam in the elevation plane. This result is expected since the space between the antenna elements exceeds $\lambda / 2$ at $72.5 \mathrm{GHz}$. On the other hand, due to the offset introduced in the azimuth plane, the grating lobe for the $40^{\circ}$ beam-steering angle is not higher than the main beam. As can be seen from Figure 18, beam steering also works well in the azimuth plane.

\section{Discussion}

This kind of antenna concept could be utilized in 5G millimeter-wave communications in three conditions: (1) the antenna has to be scalable for higher gains, (2) it has to be realizable with low-cost and high-volume manufacturing techniques, and (3) the phase shifters can be realized with reasonable performance and cost. The scalability will not be a problem once the power division network can be reliably manufactured. For example, one can easily scale the proposed antenna array into $8 \times 8$ by integrating four $4 \times 4$ antenna arrays with an additional one-to-four waveguide feed network. Injection molding could provide the low-cost technology for manufacturing such antennas. For the phase shifters, there is a lot of recent and ongoing research indicating that suitable small-sized active components will be available in the near future $[24,25]$. 


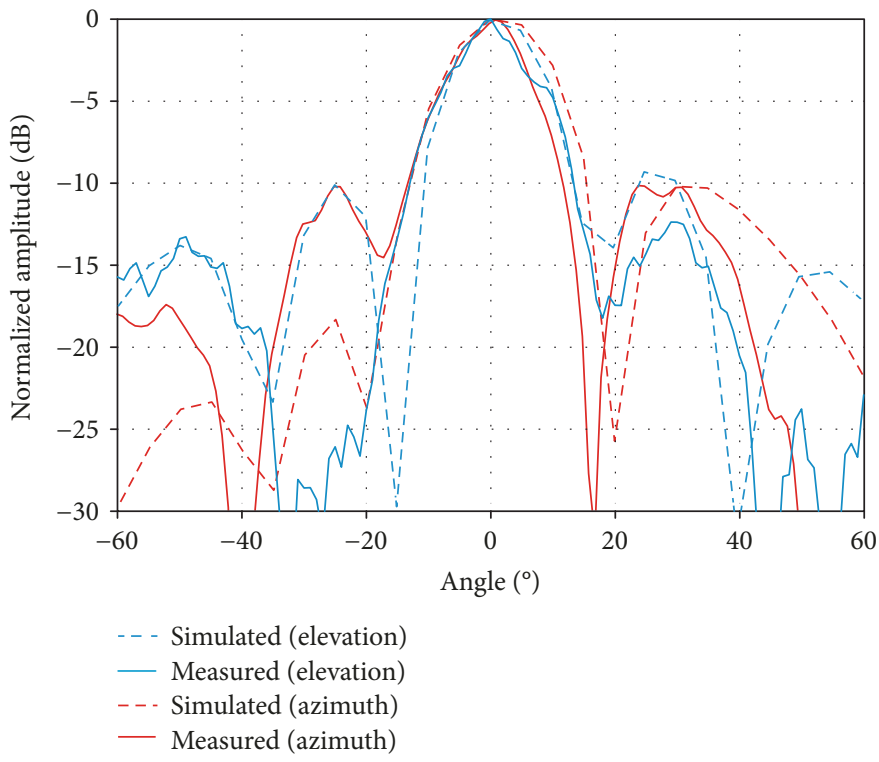

Figure 15: Measured and simulated normalized radiation pattern cuts for the antenna positioning towards the boresight direction at $72.5 \mathrm{GHz}$.

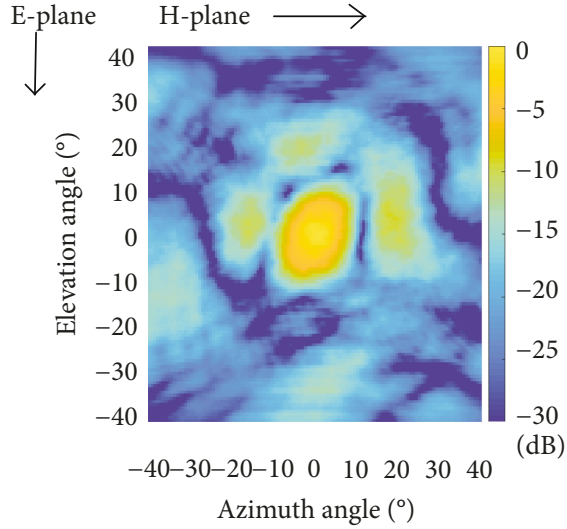

(a)

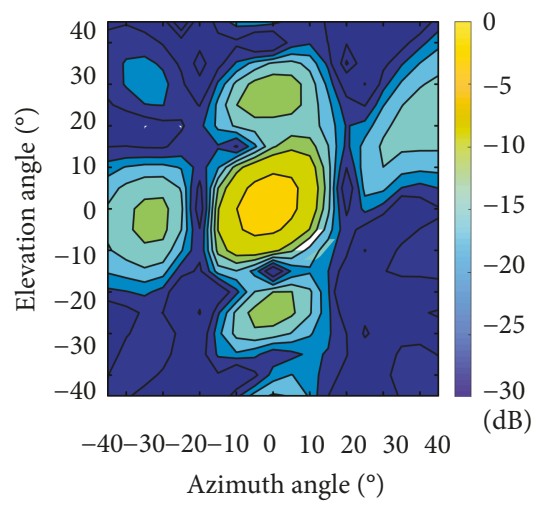

(b)

FIGURE 16: Measured and simulated normalized 2D radiation patterns for the antenna pointing towards the boresight direction at $72.5 \mathrm{GHz}$.

TABLE 3: Simulated and measured antenna characteristics for different beam-steering angles at $72.5 \mathrm{Ghz}$.

\begin{tabular}{lcccc}
\hline PCB & Angle & $\begin{array}{c}\text { Gain sim./ } \\
\text { meas. }(\mathrm{dBi})\end{array}$ & $\begin{array}{c}3 \mathrm{~dB} \text { angular } \\
\text { width sim./meas. }\end{array}$ & $\begin{array}{c}\text { Efficiency } \\
\text { meas. }(\mathrm{dB})\end{array}$ \\
\hline Boresight & & $16.9 / 15.2$ & $18.6^{\circ} / 18.4^{\circ}$ & -3.6 \\
& $10^{\circ}$ & $14.2 / 13.3$ & $19.2^{\circ} / 18.6^{\circ}$ & -3.3 \\
Elevation & $20^{\circ}$ & $13.8 / 13.1$ & $19.8^{\circ} / 18.2^{\circ}$ & -3.8 \\
plane & $30^{\circ}$ & $12.7 / 12.1$ & $21^{\circ} / 20.8^{\circ}$ & -3.5 \\
& $40^{\circ}$ & $11.7 / 11.2$ & $22.7^{\circ} / 21.6^{\circ}$ & -3.6 \\
& $10^{\circ}$ & $16.7 / 15.1$ & $19.8^{\circ} / 18.7^{\circ}$ & -3.6 \\
Azimuth & $20^{\circ}$ & $15.7 / 13.7$ & $19.1^{\circ} / 18.8^{\circ}$ & -3.2 \\
plane & $30^{\circ}$ & $13.2 / 11.6$ & $21.6^{\circ} / 20.8^{\circ}$ & -3.4 \\
& $40^{\circ}$ & $12 / 11.3$ & $22.1^{\circ} / 21.8^{\circ}$ & -3.4 \\
Diagonal & $10^{\circ}$ & $15.4 / 13.6$ & $18.8^{\circ} / 18.5^{\circ}$ & -3.6 \\
direction & $20^{\circ}$ & $14.8 / 13.2$ & $18.9^{\circ} / 18.3^{\circ}$ & -3.9 \\
\hline
\end{tabular}

The beam-steering properties of this kind of an antenna can be utilized for example in $5 \mathrm{G} \mathrm{mm}$-wave wireless backhaul or mm-wave access point antennas. In the case of limited beam-steering ranges ( \pm 5 degrees), the fine adjustment of a point-to-point link beam could be used. The side lobes can be suppressed by proper tapering of the power distribution in the antenna. On the other hand, the concept with a wide beam-steering range could be used in a wireless backhaul self-organizing network. It would provide diversity in signal routing possibilities and robustness against line-ofsight blockage in the case of street-level small cells. In the case of wireless mobile access, the beam-steerable base station antenna would provide high gain towards the user equipment, enabling larger cell coverage and high data rates. The mitigation of side lobes and grating lobes is essential in reaching these targets. The use of active, vector modulator-based phase shifters could enable improving the radiation properties of the antenna. These components would allow also 


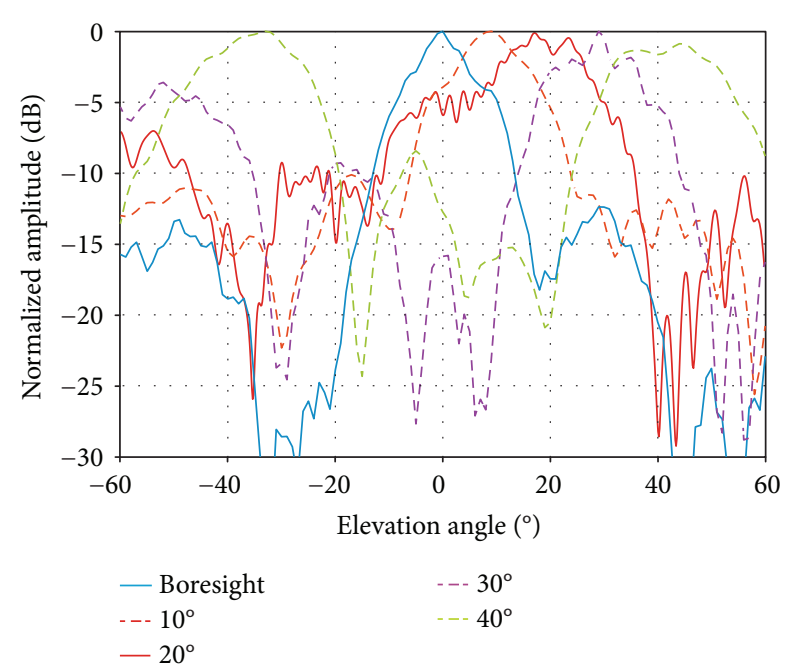

FIGURE 17: The measured normalized radiation patterns for beamsteering angles in the elevation plane.

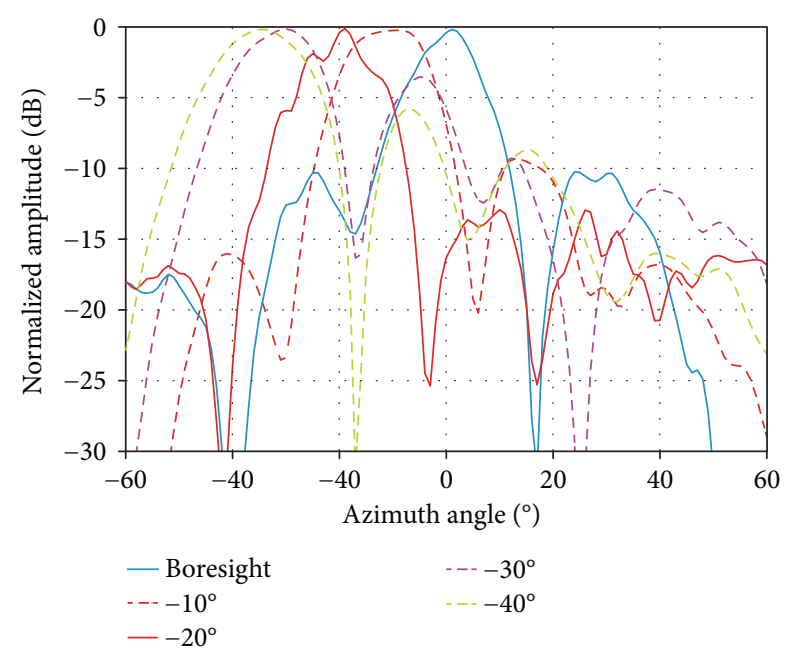

FIGURE 18: The measured normalized radiation patterns for beamsteering angles in the azimuth plane.

amplitude tapering to minimize the sidelobe level. For the grating lobe mitigation, potential methods include dielectric-filled waveguides to decrease the horn aperture size allowing $\lambda / 2$ spacing or using an irregular grid spacing of the horn elements [31].

\section{Conclusion}

A low-profile, scalable, and beam-steerable phased antenna array has been designed for the lower E-band $(71-76 \mathrm{GHz})$. The novel antenna concept combines an easy integration of phase shifters on PCB and a low-loss waveguide feed network. Beam-steering properties of the phased antenna array are demonstrated at $72.5 \mathrm{GHz}$. The designed antenna array consists of a waveguide feeding network dividing the power equally from the input waveguide to sixteen output waveguides. The PCB is used to implement the microstrip delay- line phase shifter, as well as waveguide-to-PCB transitions to connect the feeding network to the phase shifter and further to the radiating horn antenna array. In this work, fixed phase shifters are used; however, these could be replaced by active ones using flip-chipped or wire-bonded phase shifters. The antenna array is fabricated, and its operation is tested with near-field measurements. Measured radiation patterns demonstrate beam steering of $40^{\circ}$ at $72.5 \mathrm{GHz}$ in both azimuth and elevation planes. However, due to the large element spacing grating lobes appear at the largest beamsteering angles. The results are very promising and suggest that it is possible to combine active components with plastic injection moldable antennas to enable low-cost beamsteerable phased arrays.

\section{Data Availability}

The simulation and measurement data used to support the findings of this study are available from the corresponding author upon request.

\section{Conflicts of Interest}

The authors declare that they have no conflicts of interest.

\section{Acknowledgments}

The authors would like to thank Mr. Eino Kahra for preparing drawings for the antenna manufacturing. This research was supported in part by the Nokia Bell Labs. The work of M. M. Islam was supported by the Nokia Foundation.

\section{References}

[1] J. G. Andrews, S. Buzzi, W. Choi et al., "What will 5G be?," IEEE Journal on Selected Areas in Communications, vol. 32, no. 6, pp. 1065-1082, 2014.

[2] Z. Pi and F. Khan, "An introduction to millimeter-wave mobile broadband systems," IEEE Communications Magazine, vol. 49, no. 6, pp. 101-107, 2011.

[3] A. Ghosh, T. A. Thomas, M. C. Cudak et al., "Millimeter-wave enhanced local area systems: a high-data-rate approach for future wireless networks," IEEE Journal on Selected Areas in Communications, vol. 32, no. 6, pp. 1152-1163, 2014.

[4] T. S. Rappaport, R. W. Heath Jr., R. C. Daniels, and J. N. Murdock, Millimeter Wave Wireless Communications, Prentice-Hall, Upper Saddle River, NJ, USA, 2014.

[5] M. Shariat, M. Dianati, K. Seppänen, T. Suihko, J. Putkonen, and V. Frascolla, "Enabling wireless backhauling for next generation mmWave networks," in 2015 European Conference on Networks and Communications (EuCNC), pp. 164-168, Paris, France, June-July 2015.

[6] T. S. Rappaport, Shu Sun, R. Mayzus et al., "Millimeter wave mobile communications for 5G cellular: it will work!," IEEE Access, vol. 1, pp. 335-349, 2013.

[7] J. Ala-Laurinaho, J. Aurinsalo, A. Karttunen et al., "2-D beamsteerable integrated lens antenna system for $5 \mathrm{G}$ E-band access and backhaul," IEEE Transactions on Microwave Theory and Techniques, vol. 64, no. 7, pp. 2244-2255, 2016.

[8] J. Ala-Laurinaho, A. Karttunen, and A. V. Räisänen, "A mmwave integrated lens antenna for E-band beam steering," in 
2015 9th European Conference on Antennas and Propagation (EuCAP), pp. 1-2, Lisbon, Portugal, April 2015.

[9] W. Rotman and R. Turner, "Wide-angle microwave lens for line source applications," IEEE Transactions on Antennas and Propagation, vol. 11, no. 6, pp. 623-632, 1963.

[10] K. Wincza, K. Staszek, I. Slomian, and S. Gruszczynski, "Scalable multibeam antenna arrays fed by dual-band modified Butler matrices," IEEE Transactions on Antennas and Propagation, vol. 64, no. 4, pp. 1287-1297, 2016.

[11] J. Säily, M. Pokorný, M. Kaunisto, A. Lamminen, J. Aurinsalo, and Z. Raida, "Millimetre-wave beamswitching Rotman lens antenna designs on multi-layered LCP substrates," in 2016 10th European Conference on Antennas and Propagation (EuCAP), pp. 1-5, Davos, Switzerland, April 2016.

[12] B. Mencagli, R. V. Gatti, L. Marcaccioli, and R. Sorrentino, "Design of large mm-wave beam-scanning reflectarrays," in The European Conference on Wireless Technology, 2005, pp. 475-478, Paris, France, October 2005.

[13] A. Tamminen, S. Mäkelä, J. Ala-Laurinaho et al., "Reflectarray design for 120-GHz radar application: measurement results," IEEE Transactions on Antennas and Propagation, vol. 61, no. 10, pp. 5036-5047, 2013.

[14] S. Dieter, P. Feil, and W. Menzel, "Folded reflectarray antenna using a modified polarization grid for beam-steering," in Proceedings of the 5th European Conference on Antennas and Propagation (EUCAP), pp. 1400-1403, Rome, Italy, April 2011.

[15] N. Kundtz and D. R. Smith, "Extreme-angle broadband metamaterial lens," Nature Materials, vol. 9, no. 2, pp. 129-132, 2010.

[16] J. Hunt, N. Kundtz, N. Landy et al., "Broadband wide angle lens implemented with dielectric metamaterials," Sensors, vol. 11, no. 8, pp. 7982-7991, 2011.

[17] F. Sun, Y. Ma, and S. He, "Two beam steering lenses enabled by metamaterials," in 2015 IEEE MTT-S International Microwave Workshop Series on Advanced Materials and Processes for RF and THz Applications (IMWS-AMP), pp. 1-3, Suzhou, China, July 2015.

[18] Z. N. Wu, W. X. Tang, and T. J. Cui, "A beam-steerable metamaterial lens using varactor diodes," in 2015 IEEE MTT-S International Microwave Workshop Series on Advanced Materials and Processes for $\mathrm{RF}$ and THz Applications (IMWSAMP),, pp. 1-3, Suzhou, China, July 2015.

[19] T. Jiang, Z. Wang, D. Li et al., "Low-DC voltage-controlled steering-antenna radome utilizing tunable active metamaterial," IEEE Transactions on Microwave Theory and Techniques, vol. 60, no. 1, pp. 170-178, 2012.

[20] H. Li, D. Ye, F. Shen et al., "Reconfigurable diffractive antenna based on switchable electrically induced transparency," IEEE Transactions on Microwave Theory and Techniques, vol. 63, no. 3, pp. 925-936, 2015.

[21] M. Fakharzadeh, M. R. Nezhad-Ahmadi, B. Biglarbegian, J. Ahmadi-Shokouh, and S. Safavi-Naeini, "CMOS phased array transceiver technology for $60 \mathrm{GHz}$ wireless applications," IEEE Transactions on Antennas and Propagation, vol. 58, no. 4, pp. 1093-1104, 2010.

[22] X. Gu, A. Valdes-Garcia, A. Natarajan, B. Sadhu, D. Liu, and S. K. Reynolds, "W-band scalable phased arrays for imaging and communications," IEEE Communications Magazine, vol. 53, no. 4, pp. 196-204, 2015.
[23] Y. Li and K.-M. Luk, "Low-cost high-gain and broadband substrate-integrated-waveguide-fed patch antenna array for 60GHz band," IEEE Transactions on Antennas and Propagation, vol. 62, no. 11, pp. 5531-5538, 2014.

[24] Y. Yu, P. G. M. Baltus, A. de Graauw, E. van der Heijden, C. S. Vaucher, and A. H. M. van Roermund, "A $60 \mathrm{GHz}$ phase shifter integrated with LNA and PA in $65 \mathrm{~nm}$ CMOS for phased array systems," IEEE Journal of Solid-State Circuits, vol. 45, no. 9, pp. 1697-1709, 2010.

[25] F. Meng, K. Ma, K. S. Yeo, and S. Xu, "A 57-to-64-GHz 0.094$\mathrm{mm}^{2}$ 5-bit passive phase shifter in 65-nm CMOS," IEEE Transactions on Very Large Scale Integration (VLSI) Systems, vol. 24, no. 5, pp. 1917-1925, 2016.

[26] Y. Konishi, T. Nishino, H. Yukawa, and Y. Aramaki, "Millimeter-wave plastic waveguide phased array antenna," in 2010 IEEE Antennas and Propagation Society International Symposium, pp. 1-4, Toronto, ON, Canada, July 2010.

[27] R. Dolp, W. Mayer, and W. Grabherr, "Industrialization of a $58 \mathrm{GHz}$ high gain flat panel antenna design using injection molding technique," in 1999 29th European Microwave Conference, vol. 3, pp. 13-15, Munich, Germany, October 1999.

[28] T. Sehm, A. Lehto, and A. V. Raisanen, "A large planar 39$\mathrm{GHz}$ antenna array of waveguide-fed horns," IEEE Transactions on Antennas and Propagation, vol. 46, no. 8, pp. 11891193, 1998.

[29] T. Sehm, A. Lehto, and A. V. Raisanen, "A high-gain 58-GHz box-horn array antenna with suppressed grating lobes," IEEE Transactions on Antennas and Propagation, vol. 47, no. 7, pp. 1125-1130, 1999.

[30] HUBER+SUHNER, SENCITY Matrix 70/80 GHz Antenna 38 $d B i, 2018,1378.99 .0001$ datasheet.

[31] H. Wang, D. G. Fang, and Y. L. Chow, "Grating lobe reduction in a phased array of limited scanning," IEEE Transactions on Antennas and Propagation, vol. 56, no. 6, pp. 1581-1586, 2008. 


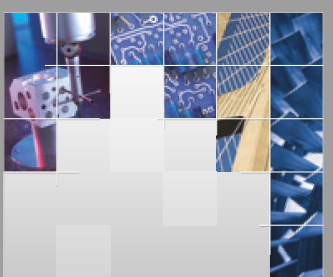

\section{Enfincering}
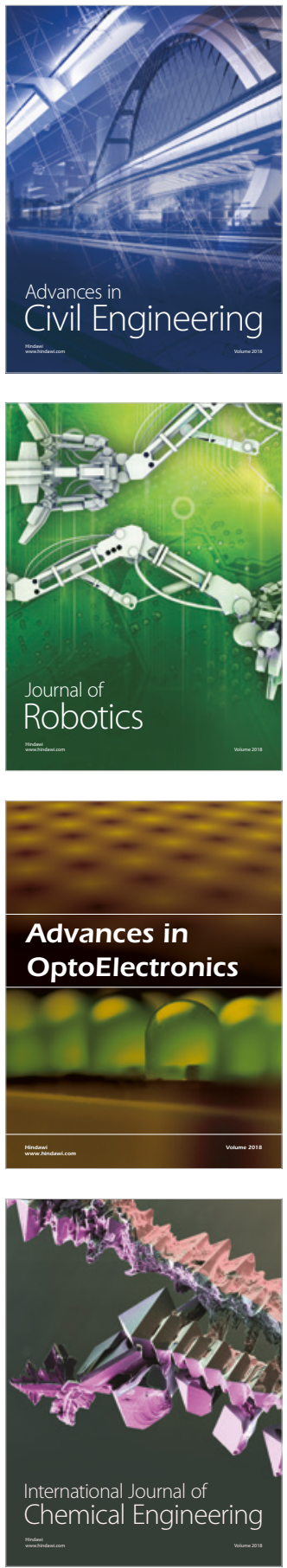

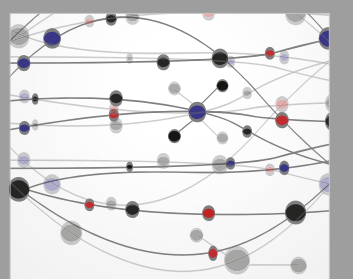

\section{Rotating \\ Machinery}

The Scientific World Journal

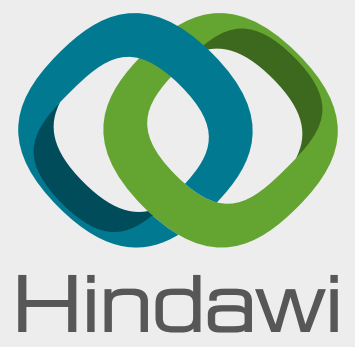

Submit your manuscripts at

www.hindawi.com
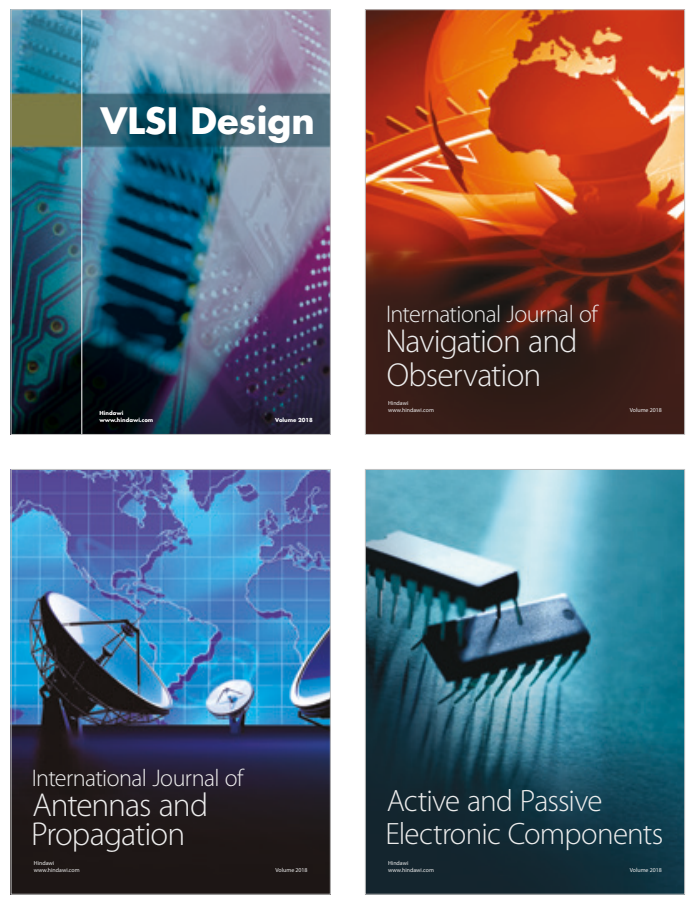
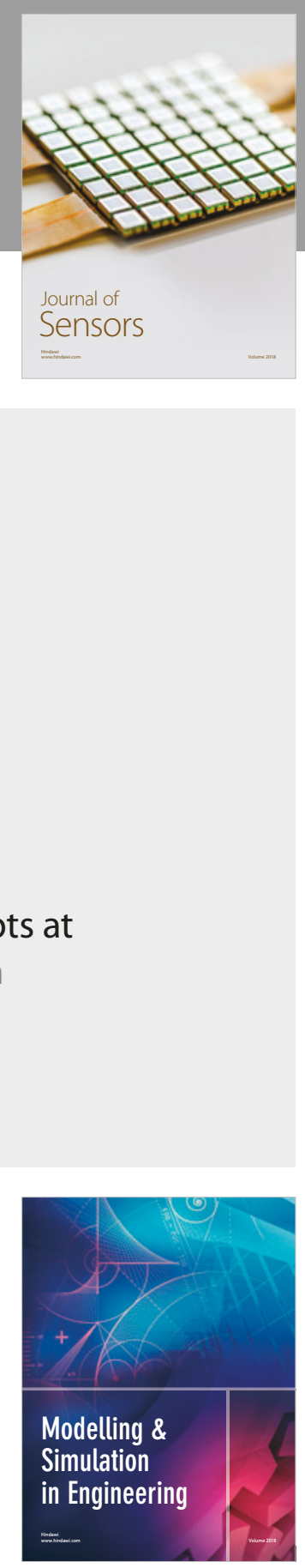

\section{Advances \\ Multimedia}
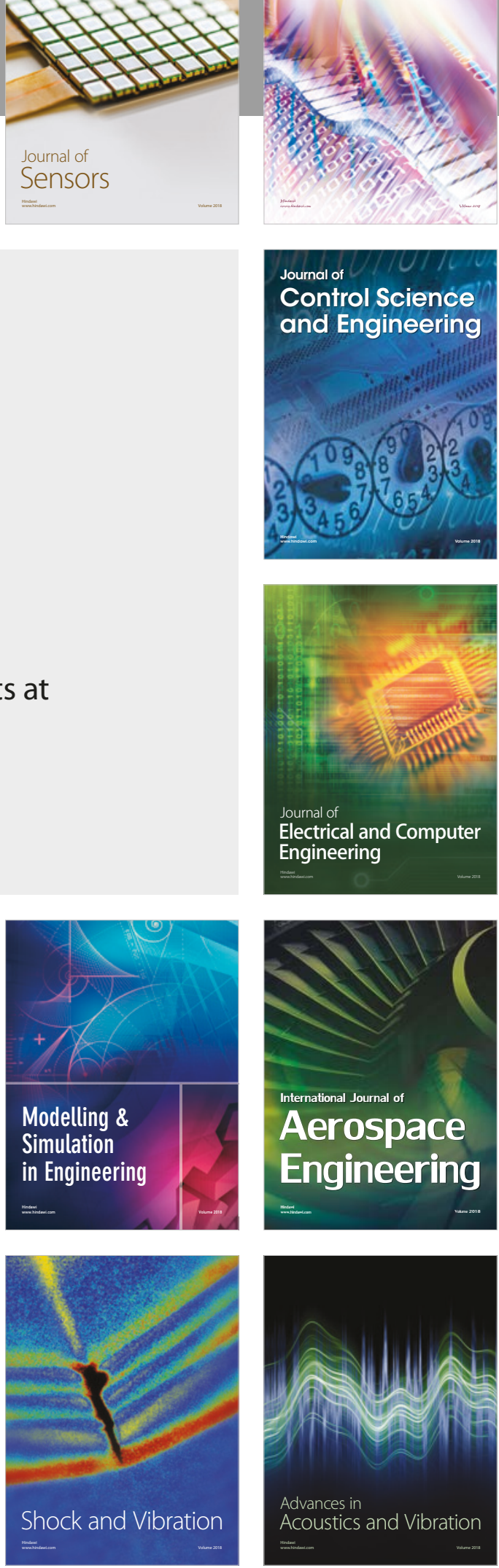